\title{
Mouse sperm capacitation in vitro involves loss of a surface-associated inhibitory component
}

\author{
Lynn R. Fraser \\ Human Biology Department, Chelsea College, Manresa Road, London SW3 6LX, U.K.
}

\begin{abstract}
Summary. The increasing fertility of epididymal mouse sperm suspensions as preincubation time is extended is accompanied by the inactivation or destruction of an inhibitory component. Alternatively, precocious removal of the component, achieved by centrifugation, leads to significant improvement in fertilizing ability. Suspensions were preincubated for a total of $120 \mathrm{~min}$, with aliquants being removed at 5, 30 and 120 min. By gently washing samples and resuspending in fresh medium, the poor fertility of unwashed 5- and 30-min suspensions was increased such that 30-min washed samples did not differ significantly from fully capacitated, highly fertile 120 -min unwashed control samples. When the supernatants obtained during washing of uncapacitated suspensions ( 5 and $30 \mathrm{~min}$ preincubation) were added to capacitated (120 min preincubation) populations, fertilization of cumulus-intact eggs was markedly and significantly inhibited, although fertilization of zona-free eggs was unaffected. In contrast, supernatants from capacitated suspensions were not inhibitory. When suspensions were preincubated in $\mathrm{Ca}^{2+}$-free media, both washing and exposure to hyperosmolal conditions improved fertilizing ability after addition of exogenous $\mathrm{Ca}^{2+}$, although not to the extent seen in control samples. Removal of the inhibitory component therefore increased the response of spermatozoa to $\mathrm{Ca}^{2+}$. The component was shown to be cell-associated and to inhibit the acrosome reaction in capacitated suspensions. Finally, the inhibition was shown to be reversible, with further incubation of inhibited suspensions restoring the original fertility.
\end{abstract}

\section{Introduction}

The spermatozoa released from the male tract of most mammals are not immediately fertile. Rather, there is a species-dependent interval during which spermatozoa acquire the ability to fertilize eggs, this modification to a potentially fertilizing state being termed capacitation (Austin, 1951; Chang, 1951). Capacitation appears to involve functional changes in the spermatozoa which prepare them to undergo the acrosome reaction (Bedford, 1970) and promote alterations in their motility patterns (e.g. Yanagimachi, 1970; Fraser, 1977), both of which are required for successful fertilization of recently ovulated eggs (Yanagimachi, 1981).

The absence of major ultrastructural alterations which distinguish capacitated from uncapacitated cells suggests that more subtle changes are involved. Chang's observation (1957) that introduction of seminal plasma caused a reversible inhibition of fertilizing ability of capacitated spermatozoa, and the demonstration by Bedford \& Chang (1962) that the inhibitory 'decapacitation factor' could be removed by centrifugation, have led to the general conclusion that capacitation involves removal of molecules from the sperm surface. Such loss of surface-associated components, of epididymal and seminal plasma origin, has been detected in a number of studies (reviewed by Johnson, 1975; O'Rand, 1979, 1982), although the various decapacitating factors reported appear to differ in their mechanism(s) of action. The fact that brief exposure of sperm suspensions to elevated ionic strength conditions, which can cause loss of surface-associated 
molecules, often enhances the demonstrable fertility of suspensions in vitro (see Oliphant \& Brackett, 1973; Brackett \& Oliphant, 1975; Fraser, 1983b) is consistent with this hypothesis.

The present experiments examined whether capacitation of epididymal mouse spermatozoa in vitro involves the loss of such a cell-associated component.

\section{Materials and Methods}

Media. A modified Tyrode's solution (124.54 mm-NaCl, $25.00 \mathrm{~mm}-\mathrm{NaHCO}_{3}, 2.68 \mathrm{~mm}-\mathrm{KCl}, 0.36$ mM-NaH ${ }_{2} \mathrm{PO}_{4}, 0.49 \mathrm{~mm}-\mathrm{MgCl}_{2}, 5.56 \mathrm{~mm}$-glucose, $1.80 \mathrm{~mm}-\mathrm{CaCl}_{2}$ and 100 units sodium penicillin/ml) containing BSA ( $4 \mathrm{mg} / \mathrm{ml}$; Armour, Eastbourne, Sussex, U.K.) was used in most experiments. In a few experiments, $\mathrm{CaCl}_{2}(1.8 \mathrm{mM})$ was omitted from the medium but because the difference in osmolality was slight, no further adjustments were made. Traces of calcium present in the other Analar-grade reagents contributed a maximum of $0.01 \mathrm{mM}$ and such media fail to support fertilization in this system (Fraser, 1982). To add 1.8 $\mathrm{mM}^{-\mathrm{Ca}^{2}}{ }^{+}$to sperm supernatants (Series V and VI), $25 \mu \mathrm{l}$ of a $\times 20$ stock solution of $\mathrm{CaCl}_{2}(36 \mathrm{mM})$ in basic medium were added per $0.5 \mathrm{ml} \mathrm{Ca}^{2+}$ free medium. In one series, $\mathrm{Ca}^{2+}$ hyperosmolal medium containing $25 \mathrm{~mm}$ additional $\mathrm{NaCl}$ was also used.

In-vitro fertilization. Mature TO female mice ( $>8$ weeks) were induced to superovulate by i.p. injections of 7.5 i.u. PMSG (Gestyl: Organon, Morden, Surrey, U.K.) and, after $48-54$ h, 5 i.u. hCG (Pregnyl: Organon). Unfertilized, cumulus-intact eggs, recovered $14 \mathrm{~h}$ after hCG injection, were released directly into sperm suspensions. Zona-free eggs were prepared by sequential treatment with hyaluronidase and pronase to remove cumulus cells and zonae, respectively, as described by Fraser (1983a). When sperm suspensions were preincubated for various times and tested for fertilizing ability, hCG was injected asynchronously so that all eggs used at any single time were recovered $14 \mathrm{~h}$ after hCG.

Epididymal sperm suspensions were prepared by releasing the contents of 1 cauda epididymidis from each of 2 mature ( $>8$ weeks) TO male mice into $1 \mathrm{ml}$ medium. Suspensions were preincubated for a total of $120 \mathrm{~min}$; in some experiments aliquants were removed after 5 and $30 \mathrm{~min}$ and washed as detailed below or left unwashed. Samples were then diluted 10 -fold to give a concentration of $1-2 \times 10^{6}$ cells $/ \mathrm{ml}$ and eggs were released into $300 \mu$ droplets of these suspensions.

All incubations were carried out in 30-mm plastic culture dishes (Sterilin, Teddington, Middx, U.K.) at $37^{\circ} \mathrm{C}$; droplets of medium were overlaid with sterile (autoclaved) liquid paraffin (Boots, Nottingham, U.K.). The gas phase used for incubations was $5 \% \mathrm{CO}_{2}-5 \% \mathrm{O}_{2}-90 \% \mathrm{~N}_{2}$.

Washing of sperm suspensions. In several experimental series, sperm suspensions were washed by gently mixing 1 volume of suspension with 2 volumes of medium (dilution factor of $1 / 3$ ) and then centrifuging at $750 \mathrm{~g}$ (room temperature) for $5 \mathrm{~min}$; the supernatant was removed and the pellet resuspended to the original volume of suspension.

Preparation of sperm supernatants. In some experiments, the supernatants obtained during washing were centrifuged for $4 \mathrm{~min}$ at $11600 \mathrm{~g}$ (room temperature) to remove cells not pelleted by the gentle centrifugation used for washing. In other experiments, supernatants were prepared directly by mixing 1 volume of suspension with 1 or 2 volumes of medium as noted (dilution factor of $1 / 2$ or $1 / 3$, respectively) and centrifuging at $11600 \mathrm{~g}$ as above. In all instances, the resulting cellfree supernatant was used.

Preparation of sperm samples for electron microscopy. Sperm samples were fixed by adding an equal volume of $5 \%$ glutaraldehyde in $0 \cdot 1 \mathrm{M}$-cacodylate buffer, $\mathrm{pH} 7 \cdot 4$. After centrifugation at $11600 \mathrm{~g}$, the supernatant was removed and fresh $2.5 \%$ glutaraldehyde in buffer was added. After fixation overnight in the cold, the cell pellets were washed without resuspension in buffer, post- 
fixed in $1 \%$ osmium tetroxide, dehydrated in graded alcohols and embedded in Spurr's resin. Ultrathin sections were stained with uranyl acetate and lead citrate before examination. The proportions of spermatozoa with and without acrosomes were assessed, with $250-300$ cells being counted in each sample.

Routine assessments. At 65-70 min after mixing eggs and spermatozoa, eggs were transferred to small droplets of fresh medium; at $75 \mathrm{~min}$, all dishes were flooded with neutral buffered formalin ( $4 \%$ formaldehyde). Eggs were then stained and mounted as described by Fraser (1983a). Eggs were considered to be fertilized if they had resumed meiosis II and contained a decondensing sperm head. The rate of sperm penetration was assessed by determining the proportion of fertilized eggs that exhibited maximal nuclear development for the 75-min interval, i.e. those eggs that had reached the telophase-second polar body stage and possessed a fully decondensed sperm head (see Fraser, 1983a).

Preincubated and diluted sperm suspensions were assessed for expression of hyperactivated motility, a specific motility pattern associated with mouse sperm capacitation and hence fertilizing ability (Fraser, 1977).

Cochran's test for the combination of $2 \times 2$ contingency tables (Snedecor \& Cochran, 1967) was used to analyse results. This permits comparisons within individual experiments and allows for variations amongst different sperm suspensions as well as differences in sample sizes.

\section{Results}

Series I: effects of washing sperm suspensions on fertilization and rate of sperm penetration

Sperm suspensions were tested for fertilizing ability after 5, 30 and 120 min preincubation in control medium. At 5 and $30 \mathrm{~min}$, two aliquants were removed and one was washed as detailed earlier. Washed and unwashed samples were diluted and cumulus-intact eggs were added. Only unwashed samples were examined at $120 \mathrm{~min}$ since preliminary experiments indicated no detectable difference between washed and unwashed samples at this time. Because washing removed the bulk of free hyaluronidase present in suspensions, cumulus dispersal from eggs was usually incomplete and a brief exposure to hyaluronidase was necessary before fixation to remove these cells which would otherwise hinder assessment. Three replicate experiments were performed $(\mathrm{N}=3)$.

Sperm suspensions preincubated for $120 \mathrm{~min}$ were highly fertile $(\sim 90 \%$ of eggs fertilized) and showed rapid rates of egg penetration, with $95 \%$ of penetrated eggs at the stage of maximal nuclear development (Table 1). In contrast, suspensions preincubated for $5 \mathrm{~min}$ were very poorly fertile. Although washed samples fertilized a significantly higher proportion of eggs than unwashed samples $(7 \%$ vs $39 \% ; P<0.05)$, both 5 -min groups promoted significantly lower levels of

Table 1. In-vitro fertilizing ability of unwashed and washed preincubated epididymal mouse sperm suspensions

\begin{tabular}{cccccc}
\hline \multirow{2}{*}{$\begin{array}{c}\text { Preincubation } \\
\text { time (min) }\end{array}$} & Washed & No. & $\%$ (range) & $\begin{array}{c}\text { Maximal nuclear } \\
\text { development }(\%) \dagger\end{array}$ \\
\cline { 3 - 5 } & - & $4 / 58$ & $6 \cdot 9^{* *}(0-18)$ & 0 \\
30 & + & $31 / 80$ & $38 \cdot 8^{*}$ & $(25-79)$ & $3 \cdot 2$ \\
& - & $31 / 59$ & $52 \cdot 5^{*}$ & $(10-88)$ & $38 \cdot 7$ \\
120 & + & $70 / 77$ & $90 \cdot 9$ & $(84-100)$ & $74 \cdot 3$ \\
& - & $43 / 48$ & $89 \cdot 6$ & $(85-94)$ & $95 \cdot 3$ \\
\hline
\end{tabular}

${ }^{*} P<0.05,{ }^{* *} P<0.01$ compared with 120 -min suspensions.

$\dagger$ Fertilized eggs at telophase-second polar body with sperm heads fully decondensed. 
fertilization than control 120-min samples (unwashed, $P<0.01$; washed, $P<0.05$ ) and few of the fertilized eggs had reached advanced nuclear stages. These same suspensions were more fertile after incubation for a further $25 \mathrm{~min}$, although considerable variation amongst experiments was noted with unwashed samples. The corresponding washed samples, however, were consistently more highly fertile $(91 \%$ of eggs fertilized $; \boldsymbol{P}<0.05$ compared with unwashed suspensions) with no significant differences between these washed suspensions and the unwashed 120-min control samples. Furthermore, the majority of fertilized eggs had reached maximal nuclear development, unlike eggs in the comparable unwashed group.

In general, $\sim 60 \%$ of spermatozoa in all suspensions were motile and, after $120 \mathrm{~min}$ preincubation, the majority of these exhibited hyperactivated motility $(+++)$. In unwashed samples, hyperactivated motility was not observed after $5 \mathrm{~min}$ and even after $30 \mathrm{~min}$ it was generally negligible ( - to,$\pm<10 \%$ of cells). In most 30 -min washed samples, however, hyperactivated motility was seen $(+$ to $++; \sim 20-40 \%$ of cells).

\section{Series $I I$ : inhibition of fertilization by sperm supernatants}

Series I and Series II experiments were carried out at the same time, with those in Series II utilizing the supernatants obtained during the washing procedure for Series I. In addition to the 5and 30 -min supernatant samples, a similar preparation was made from the 120 -min preincubated suspension (see 'Materials and Methods'). These cell-free supernatant samples and 5-fold dilutions of them (representing $1 / 3$ and $1 / 15$ dilution factors of the original suspension) were used to dilute aliquants of the 120 -min preincubated control suspensions. After $5 \mathrm{~min}$ to permit interaction between sperm cells and supernatant, cumulus-intact eggs were added $(N=3)$.

Since Series I and II experiments were performed on the same original suspensions, the highly fertile 120-min preincubated control sperm samples served for both series. The supernatants obtained after 5 and 30 min preincubation significantly inhibited the fertilizing ability of control suspensions, whether the dilution factor was $1 / 3(P<0.01$; Table 2$)$ or $1 / 15(P<0.05)$. A high degree of inhibition was consistently observed with the $1 / 3$ dilutions, whereas greater variability in inhibition was observed with the more dilute samples. In addition to inhibition of fertilization, retardation of sperm penetration was observed in the eggs that were fertilized, as indicated by the lower proportions of eggs reaching maximal nuclear development than obtained with untreated control suspensions; this retardation was most pronounced in the $30 \mathrm{~min}, 1 / 3$ dilution, samples. In contrast to the above, a slight but statistically insignificant inhibition was observed with the more concentrated 120 -min supernatant, while no inhibition was observed with the more dilute preparation. There was no detectable interference with cumulus dispersal in any group.

Table 2. Inhibition of capacitated (120-min preincubated) mouse sperm in-vitro fertilizing ability by sperm supernatants prepared after preincubation

\begin{tabular}{cccccc}
\hline \multicolumn{2}{c}{ Supernatant } & & \multicolumn{2}{c}{ Eggs fertilized } & \\
\cline { 2 - 4 } $\begin{array}{c}\text { Length of } \\
\text { preincubation (min) }\end{array}$ & $\begin{array}{c}\text { Dilution } \\
\text { factor }\end{array}$ & & No. & $\%$ (range) & $\begin{array}{c}\text { Maximal nuclear } \\
\text { development (\%) }\end{array}$ \\
\hline 5 & $1 / 3$ & $5 / 42$ & $11 \cdot 9^{* *}(0-20)$ & $80 \cdot 0$ \\
30 & $1 / 15$ & $22 / 47$ & $46 \cdot 8^{*}(25-80)$ & $72 \cdot 7$ \\
& $1 / 3$ & $5 / 40$ & $12 \cdot 5^{* *}(7-20)$ & $40 \cdot 0$ \\
120 & $1 / 15$ & $21 / 39$ & $53 \cdot 8^{*}(18-88)$ & $80 \cdot 9$ \\
& $1 / 3$ & $37 / 55$ & $67 \cdot 3$ & $(17-100)$ & $75 \cdot 7$ \\
\hline \multirow{2}{*}{ Control } & $1 / 15$ & $46 / 52$ & $88 \cdot 5$ & $(75-100)$ & $78 \cdot 3$ \\
\hline
\end{tabular}

${ }^{*} P<0.05,{ }^{* *} P<0.01$ compared with control suspensions. 
All samples, whether or not exposed to supernatant, exhibited a high incidence of hyperactivated motility $(+++)$, indicating that inhibition of fertilization was not accompanied by a loss of this specific motility pattern.

\section{Series III: fertilization of cumulus-intact and zona-free eggs in the presence of sperm supernatants}

Because the inhibitory effects of supernatants appeared to be concentration-related, a more concentrated supernatant (dilution factor of 1/2) was prepared from 30-min preincubated sperm suspensions to assess fertilization of zona-free eggs. The supernatant and the control medium were used to dilute 120-min preincubated control suspensions and, after $5 \mathrm{~min}$, cumulus-intact and zonafree eggs were added to separate droplets $(\mathrm{N}=3)$.

When assessed with cumulus-intact eggs, samples diluted in control medium were highly fertile $(96 \%$, Table 3$)$, while aliquants exposed to supernatants were very poorly fertile $(13 \% ; P<0.01$ compared with controls). In contrast, these same samples were equally highly fertile when tested with zona-free eggs $(100 \%)$ and gave a similar incidence of polyspermy $(\sim 50 \%)$. The latter is a reflection of the proportion of 'fit' spermatozoa in a given population (Fraser, 1983a).

Series IV : fertilizing ability of spermatozoa preincubated in $\mathrm{Ca}^{2+}$-free medium and then washed before addition of exogenous $\mathrm{Ca}^{2+}$

Sperm suspensions were preincubated in $\mathrm{Ca}^{2+}$-containing control, $\mathrm{Ca}^{2+}$-free and hyperosmolal $\mathrm{Ca}^{2+}$-free media for $30 \mathrm{~min}$. To obtain the 3 suspensions required in this series, the contents of half an epididymis from each of 2 males were released into $0.5 \mathrm{ml}$ of each medium. Samples from control and iso-osmolal $\mathrm{Ca}^{2+}$-free suspensions were washed in the same medium used for incubation. Aliquants from the 2 washed and 3 unwashed suspensions were all diluted in $\mathrm{Ca}^{2+}$-containing control medium and cumulus-intact eggs were added. After dilution, osmolality in the high-salt sample was similar to that in the others. The control suspension was incubated another $90 \mathrm{~min}$ and again assessed $(\mathrm{N}=4)$.

Once again, control suspensions preincubated for $120 \mathrm{~min}$ were consistently highly fertile and penetrated eggs rapidly (Table 4 ). When assessed after only $30 \mathrm{~min}$ preincubation they were poorly fertile ( $P<0.01$ compared with 120 min samples), but washing promoted fertilization kinetics similar to those of the 120 -min control samples. Comparisons of suspensions preincubated for 30 min in $\mathrm{Ca}^{2+}$-free medium revealed that while unwashed samples fertilized no eggs, washed samples were able to fertilize at least some eggs in each experiment, albeit more slowly and erratically than either washed 30 -min or unwashed 120 -min control suspensions $(P<0 \cdot 01)$. Similar results, i.e. some fertilization, were obtained when spermatozoa were preincubated in hyperosmolal $\mathrm{Ca}^{2+}$-free medium. An earlier study has already shown that suspensions pre-

Table 3. Effect of sperm supernatant (from 30-min preincubated suspensions) on the ability of capacitated (120-min preincubation) epididymal mouse spermatozoa to fertilize zona-intact and zonafree eggs in vitro

\begin{tabular}{|c|c|c|c|c|c|}
\hline \multirow{2}{*}{$\begin{array}{l}\text { Medium for } \\
\text { fertilization }\end{array}$} & \multirow[b]{2}{*}{ \pm Zona } & \multicolumn{2}{|c|}{ Eggs fertilized } & \multirow{2}{*}{$\begin{array}{l}\text { Maximal nuclear } \\
\text { development }(\%)\end{array}$} & \multirow{2}{*}{$\begin{array}{c}\text { Incidence of } \\
\text { polyspermy (\%) }\end{array}$} \\
\hline & & No. & $\%$ (range) & & \\
\hline Control & + & $\begin{array}{l}49 / 51 \\
53 / 53\end{array}$ & $\begin{array}{cc}96 \cdot 1 & (90-100) \\
100 & \end{array}$ & $\begin{array}{l}95 \cdot 9 \\
100\end{array}$ & $\begin{array}{l}-\dagger \\
52 \cdot 8\end{array}$ \\
\hline Supernatant & $\begin{array}{l}+ \\
-\end{array}$ & $\begin{array}{r}7 / 53 \\
52 / 52\end{array}$ & $\begin{array}{l}13 \cdot 2^{* *}(6-25) \\
100\end{array}$ & $\begin{array}{l}100 \\
100\end{array}$ & $\begin{array}{c}-\dagger \\
48 \cdot 1\end{array}$ \\
\hline
\end{tabular}

** $P<0.01$ compared with appropriate control group.

$\dagger$ Not determined. 
Table 4. In-vitro fertilizing ability of unwashed and washed epididymal mouse sperm suspensions preincubated for $30 \mathrm{~min}$ in control or $\mathrm{Ca}^{2+}$-free media $\left(\mathrm{Ca}^{2+}\right.$ was present in all fertilization media)

\begin{tabular}{|c|c|c|c|c|}
\hline \multirow{2}{*}{$\begin{array}{l}\text { Preincubation } \\
\text { medium }\end{array}$} & \multirow[b]{2}{*}{ Washed } & \multicolumn{2}{|c|}{ Eggs fertilized } & \multirow{2}{*}{$\begin{array}{l}\text { Maximal nuclear } \\
\text { development }(\%)\end{array}$} \\
\hline & & No. & $\%$ (range) & \\
\hline Control & $\begin{array}{l}- \\
+\end{array}$ & $\begin{array}{l}18 / 66 \\
53 / 59\end{array}$ & $\begin{array}{ll}27 \cdot 3^{* *} & (0-71) \\
89 \cdot 8 & (75-96)\end{array}$ & $\begin{array}{l}38.9 \\
83.0\end{array}$ \\
\hline $\mathrm{Ca}^{2+}$-free & $\overline{+}$ & $\begin{array}{r}0 / 48 \\
30 / 76\end{array}$ & $\begin{array}{l}0^{* * *} \\
39 \cdot 5^{* *}(14-86)\end{array}$ & $\begin{array}{c}0 \\
10 \cdot 0\end{array}$ \\
\hline $\mathrm{Ca}^{2+}$-free, hyperosmolal & - & $38 / 89$ & $42 \cdot 7^{* *}(7-83)$ & $5 \cdot 3$ \\
\hline $\begin{array}{l}\text { Control } \\
\text { suspensions } \\
\text { (preinc. } 120 \mathrm{~min} \text { ) }\end{array}$ & - & $67 / 68$ & $98.5 \quad(95-100)$ & $97 \cdot 0$ \\
\hline
\end{tabular}

${ }^{* *} P<0.01,{ }^{* * *} P<0.001$ compared with 120 -min control suspensions.

Table 5. Inhibition of capacitated mouse sperm in-vitro fertilizing ability by sperm supernatants prepared from suspensions preincubated in control or $\mathrm{Ca}^{2+}$-free media $\left(\mathrm{Ca}^{2+}\right.$ was present in all fertilization media)

\begin{tabular}{lccccc}
\hline \multicolumn{2}{c}{ Supernatant } & & \multicolumn{2}{c}{ Eggs fertilized } & \\
\cline { 5 - 6 } $\begin{array}{c}\text { Preincubation } \\
\text { medium }\end{array}$ & $\begin{array}{c}\text { Preincubation } \\
\text { time (min) }\end{array}$ & & No. & $\%$ (range) & $\begin{array}{c}\text { Maximal nuclear } \\
\text { development (\%) }\end{array}$ \\
\hline Control & 30 & $28 / 67$ & $41 \cdot 2^{* *}(14-64)$ & $67 \cdot 9$ \\
$\mathrm{Ca}^{2+}{ }_{- \text {free }}$ & 120 & $21 / 28$ & $75 \cdot 0$ & $(55-100)$ & $42 \cdot 9$ \\
& 30 & $9 / 28$ & $32 \cdot 1^{*}(30-33)$ & $11 \cdot 1$ \\
\hline Control & 120 & & $27 / 46$ & $58 \cdot 7^{*}(38-71)$ & $51 \cdot 9$ \\
\hline suspensions & & $54 / 55$ & $98 \cdot 2$ & $(95-100)$ & $96 \cdot 3$ \\
\hline
\end{tabular}

${ }^{*} P<0.05,{ }^{* *} P<0.01$ compared with control suspensions.

incubated for $120 \mathrm{~min}$ in $\mathrm{Ca}^{2+}$-free medium are poorly fertile immediately upon the introduction of exogenous $\mathrm{Ca}^{2+}$ (Fraser, 1982).

Samples preincubated in control medium exhibited motility patterns as described in Series I, with hyperactivated motility evident in washed $30-\mathrm{min}$ and unwashed 120 -min suspensions. Cells preincubated in both $\mathrm{Ca}^{2+}$-free media exhibited only progressive motility, in which the sperm tail is relatively inflexible (Fraser, 1977) and washing did not cause an obvious, rapid expression of hyperactivated motility. This absence of hyperactivated motility would correlate with the slow penetration rate observed in these groups.

\section{Series $V$ : effect on fertilization of supernatants from $\mathrm{Ca}^{2+}$-free suspensions}

Sperm suspensions prepared from the same males were preincubated in control and $\mathrm{Ca}^{2+}$-free media for a total of $120 \mathrm{~min}$. At 30 and $120 \mathrm{~min}$, supernatant samples were prepared from both suspensions. After addition of $\mathrm{Ca}^{2+}$ to the $\mathrm{Ca}^{2+}$-free samples, all supernatants were used to dilute 120-min preincubated control suspensions to assess effects on the ability to fertilize cumulus-intact eggs $(\mathrm{N}=3)$.

As in Series II, supernatants from 30-min control samples significantly inhibited the fertilizing ability of 120 -min preincubated spermatozoa $(P<0.01$, Table 5), although the inhibitory effect 
had disappeared from supernatants by $120 \mathrm{~min}$. There was evidence, however, for a slower rate of sperm penetration in both groups. Both supernatants from $\mathrm{Ca}^{2+}$-free incubations significantly inhibited fertilization $(P<0.05)$ and also appeared to slow the rate of penetration in those eggs that possessed a decondensing sperm head. Unlike the $120-\mathrm{min}$ control medium supernatants, inhibitory effects were still observed with the corresponding $\mathrm{Ca}^{2+}$-free samples $(P<0.05)$. As in Series II, no deleterious effect on hyperactivated motility was observed in any sample.

\section{Series VI: is the inhibitory component cell-associated?}

(a) After preincubation of sperm suspensions for $30 \mathrm{~min}$ in control medium, 3 aliquants were removed, treated as below and diluted for fertilization assessment: Aliquant 1, no treatment (unwashed); Aliquant 2, centrifuged but resuspended in resulting supernatant (centrifuged); Aliquant 3, washed as described in 'Materials and Methods' $(\mathrm{N}=5)$.

As in the other series, 30-min preincubated, unwashed sperm suspensions exhibited various degrees of fertility (mean, $38.9 \%$; range $11-69 \%$ ) with only a minority of fertilized eggs containing sperm heads at late stages of decondensation (mean, 23.2\%). In contrast, the corresponding washed samples were uniformly highly fertile (mean, $94 \cdot 3 \%$; range $88-100 \%$ ) and most fertilized eggs contained fully decondensed sperm heads (mean, $96 \cdot 4 \%$ ). Centrifuged samples gave intermediate values for proportion of fertilized eggs (mean, 55.3\%; range $27-86 \%$ ) and proportion of eggs with fully decondensed sperm heads (mean, 57.2\%), suggesting that subjecting spermatozoa to centrifugal force caused loss of the inhibitory component from the cells.

(b) Sperm suspensions to be assessed were prepared in $\mathrm{Ca}^{2+}$-free medium as inactivation of the inhibitor appeared to be retarded under these conditions. After $5 \mathrm{~min}$, suspensions were washed as described earlier. The supernatant was retained and the washed cells, resuspended in fresh $\mathrm{Ca}^{2+}$-free medium, were incubated until $30 \mathrm{~min}$ after release. At this time, 2 further supernatants were prepared (all with dilution factor of 1/3) from (1) the above 5-min washed and subsequently rewashed suspension and (2) the original $\mathrm{Ca}^{2+}$-free suspension. After addition of exogenous $\mathrm{Ca}^{2+}$, these 3 supernatants ( $5 \mathrm{~min}, 30 \mathrm{~min}$ and $5-\mathrm{min}$ rewashed at $30 \mathrm{~min}$ ) were used to dilute $120-\mathrm{min}$ sperm suspensions preincubated in control, $\mathrm{Ca}^{2+}$-containing medium for assessing effects on fertilization of cumulus-intact eggs $(\mathrm{N}=3)$.

Supernatants prepared after 5 and 30 min were inhibitory $(P<0.001$; Table 6), as were those from rewashed suspensions $(P<0.05)$. Data from the third treatment group indicated release of the inhibitory component from the surface of washed spermatozoa. In 1 of the 3 replicate experiments, the control, $\mathrm{Ca}^{2+}$-containing suspension was treated in a like manner and yielded

Table 6. Inhibition of capacitated (120-min preincubated) sperm fertilizing ability by supernatants obtained from suspensions preincubated in $\mathrm{Ca}^{2+}$-free medium $\left(\mathrm{Ca}^{2+}\right.$ was present in all fertilization media)

\begin{tabular}{|c|c|c|}
\hline \multirow{2}{*}{$\begin{array}{l}\text { Supernatant } \\
\text { preincubation } \\
\text { (min) }\end{array}$} & \multicolumn{2}{|c|}{ Eggs fertilized } \\
\hline & No. & $\%$ (range) \\
\hline 5 & $1 / 45$ & $2 \cdot 2^{* * *}(0-5)$ \\
\hline 30 & $1 / 37$ & $2 \cdot 7^{* * *}(0-8)$ \\
\hline $30+$ & $37 / 85$ & $43 \cdot 5^{*} \quad(32-55)$ \\
\hline $\begin{array}{l}\text { Control } \mathrm{Ca}^{2+}- \\
\text { containing suspensions }\end{array}$ & $44 / 50$ & $(82-96)$ \\
\hline
\end{tabular}

${ }^{*} P<0.05,{ }^{* * *} P<0.001$ compared with 120 -min control.

$\dagger$ Suspensions washed at 5 min were incubated further and rewashed at $30 \mathrm{~min}$ to provide a second supernatant. 
similar results when the supernatants were assessed: 5 - and 30-min samples, $0 \%$ of eggs fertilized (total of $0 / 28$ ) and 30-min rewashed sample, $41 \%$ fertilized (12/29).

\section{Series VII: acrosome loss}

In washed suspensions. Sperm suspensions in control medium were preincubated for $30 \mathrm{~min}$. One aliquant was washed as above and then, along with an unwashed aliquant, diluted 10-fold to give a sperm concentration similar to that for fertilization experiments. Preliminary experiments evaluating samples with the light microscope indicated no detectable differences in acrosome loss immediately after washing (data not presented). Subsequent samples were incubated a further 40 min to determine whether changes in rate of acrosome loss following treatment accompanied the significant changes in fertilizing potential (e.g. Series I, Table 1). Samples were fixed, processed as above and examined with the electron microscope $(\mathrm{N}=3)$. No significant differences were detected between the 2 groups: unwashed, $33 \cdot 9 \pm 4 \cdot 2$; washed, $29.9 \pm 2.9(\%$ spermatozoa lacking acrosome \pm s.e.m.). There was no evidence that washing per se damaged the acrosome or other structures in the spermatozoa.

In the presence of sperm supernatants. Sperm suspensions were preincubated for $120 \mathrm{~min}$ in control medium and then diluted 10 -fold in control medium or supernatant (dilution factor of $1 / 2$ ) prepared from $30-\mathrm{min}$ preincubated suspensions. After incubation for a further 40 min during which time acrosome loss would be expected to continue in control samples, samples were fixed, processed and assessed with the electron microscope. In one experiment, an aliquant of the sample was fixed immediately after dilution in control medium $(\mathrm{N}=3)$.

Acrosome loss was significantly inhibited $(P<0.01)$ in the presence of sperm supernatants, compared with comparable samples incubated in control medium (Table 7). The results obtained in the immediately fixed sample suggest that essentially all subsequent acrosome loss was inhibited in the presence of supernatants.

\section{Series VIII: reversibility of inhibition}

Because appreciable acrosome loss had occurred in capacitated suspensions preincubated for $120 \mathrm{~min}$ and this might interfere with 'recapacitation' after introduction of the inhibitory component, suspensions washed after $30 \mathrm{~min}$ preincubation, being highly fertile while retaining their acrosomes, were used to assess possible reversibility. To make test conditions as similar as possible to those found in an uncapacitated suspension, a supernatant was prepared without dilution from a sperm suspension preincubated for $15 \mathrm{~min}$. A second suspension was preincubated for $30 \mathrm{~min}$; 2 aliquants were removed, washed and resuspended, one in supernatant and the other in control medium. After $10 \mathrm{~min}$, samples from the 2 washed suspensions and the original, unwashed

Table 7. Acrosome loss in mouse sperm suspensions preincubated for $120 \mathrm{~min}$ in control medium, then diluted into control medium or sperm supernatant (from 30-min preincubation samples) and incubated for another $40 \mathrm{~min}$

\begin{tabular}{|c|c|c|}
\hline \multirow[b]{2}{*}{ Sample no. } & \multicolumn{2}{|c|}{ Acrosome-free spermatozoa $(\%)$} \\
\hline & Control medium & Epididymal supernatant \\
\hline 1 & $64.6(52.0) \dagger$ & $46 \cdot 9$ \\
\hline 2 & $54 \cdot 3$ & $37.0\}^{* *}$ \\
\hline 3 & 57.4 & $38.4 J$ \\
\hline
\end{tabular}

** $P<0.01$ compared with control samples.

$\dagger$ Sample fixed at time of dilution. 
Table 8. Reversibility of inhibition induced by sperm supernatants

\begin{tabular}{|c|c|c|c|c|c|c|}
\hline \multirow{4}{*}{$\begin{array}{l}\text { Treatment at } \\
30 \mathrm{~min}\end{array}$} & \multicolumn{6}{|c|}{ Total length of preincubation } \\
\hline & \multicolumn{3}{|c|}{$30 \mathrm{~min}$} & \multicolumn{3}{|c|}{$150 \mathrm{~min}$} \\
\hline & \multicolumn{2}{|c|}{ Eggs fertilized } & \multirow{2}{*}{$\begin{array}{c}\text { Maximal } \\
\text { nuclear } \\
\text { development }(\%)\end{array}$} & \multicolumn{2}{|c|}{ Eggs fertilized } & \multirow{2}{*}{$\begin{array}{c}\text { Maximal } \\
\text { nuclear } \\
\text { development }(\%)\end{array}$} \\
\hline & No. (range) & $\%$ & & No. (range) & $\%$ & \\
\hline None (control) & $18 / 74(14-32)$ & $24 \cdot 3^{*}$ & $11 \cdot 1$ & $50 / 58(77-92)$ & $86 \cdot 2$ & $94 \cdot 0$ \\
\hline $\begin{array}{l}\text { Wash, resuspended in } \\
\text { control medium }\end{array}$ & $57 / 70(81-83)$ & $81 \cdot 4$ & $81 \cdot 0$ & $82 / 91(86-94)$ & $90 \cdot 1$ & 97.6 \\
\hline $\begin{array}{l}\text { Wash, resuspended in } \\
\text { sperm supernatant }\end{array}$ & $35 / 124(18-38)$ & $28 \cdot 2^{*} \dagger$ & $14 \cdot 3$ & $102 / 119(57-100)$ & $85 \cdot 7$ & $95 \cdot 1$ \\
\hline
\end{tabular}

${ }^{*} P<0.05$ compared with $30-\mathrm{min}$ washed and $150-\mathrm{min}$ unwashed and washed samples.

$\dagger P<0.05$ compared with 150 -min washed sample resuspended in supernatant at $30 \mathrm{~min}$.

suspension were removed, diluted in control medium and eggs were added. The remainder of the washed and unwashed suspensions was incubated further and tested again. In the first replicate, the second incubation was for $90 \mathrm{~min}$, while in the next 2 it was $120 \mathrm{~min}$ for a total incubation time of $150 \mathrm{~min}$; results have been combined in Table $8(\mathrm{~N}=3)$.

As noted in the earlier series, unwashed 30 -min suspensions were poorly fertile (Table 8 ). The washed suspensions were significantly more fertile $(P<0.05)$, while the washed samples resuspended in sperm supernatant were poorly fertile $(P<0.05$, compared with washed samples in control medium). After the extended preincubation, all suspensions were highly fertile and no significant differences could be detected amongst them, indicating that the inhibition caused by experimental treatment was reversible. The $150-\mathrm{min}$ total incubation promoted a higher rate of fertilization ( 80 and $100 \%)$ than did the 120 -min incubation $(57 \%$ ), suggesting that about $120 \mathrm{~min}$ is required to complete the recapacitation process.

\section{Discussion}

These experiments indicate that capacitation in vitro of epididymal spermatozoa involves the timedependent destruction or inactivation of an inhibitory factor. Removal of the active component(s) from uncapacitated suspensions by centrifugation and resuspension in fresh medium leads to an immediate increase in fertilizing ability, as determined both by a significant increase in the proportion of eggs fertilized and more rapid sperm penetration into these eggs. In contrast, exposure of demonstrably fertile capacitated spermatozoa to sperm supernatants quickly renders them poorly fertile. Supernatants prepared from essentially uncapacitated suspensions (e.g. 5 and 30 min preincubation) proved to be highly inhibitory, even after considerable dilution, while supernatants from capacitated suspensions (120 min preincubation) failed to produce statistically significant inhibition. Thus the decrease in inhibitory activity accompanies the increase in fertilizing ability of unwashed epididymal suspensions. A comparable decrease in ejaculated sperm samples can be inferred from the similarity in fertilization kinetics observed with epididymal and uterine sperm populations (Fraser, 1983b). The inhibition induced by introducing the inhibitory component to highly fertile suspensions was shown to be reversible, with fertility returning over the same time course observed during capacitation of unmanipulated suspensions.

That at least some of the inhibitory component is sperm-associated was demonstrated by two experimental approaches. With the first, simply exposing spermatozoa to centrifugation and resuspension without subsequently removing epididymal fluid generally increased the fertilizing 
ability of suspensions to levels intermediate between those of washed and unwashed suspensions; this procedure presumably dissociated the component from the sperm surface sufficiently to permit fertilization before reassociation occurred. With the second approach, freshly prepared suspensions were washed carefully to remove any soluble inhibitor molecules, incubated further and then rewashed. The inhibitory ability of supernatants from rewashed suspensions indicates that the cells themselves were the source of the inhibitor.

In considering the mechanism of action, two possible specific sites of action are the acrosome and the tail. Results in this study clearly indicate that washing sperm suspensions which are demonstrably poorly functional will promote optimal function in most instances. Since available evidence indicates that only acrosome-reacted spermatozoa can fertilize eggs (Yanagimachi, 1981), it might be inferred that an increase in acrosome loss accompanies this increased fertility. However, electron microscopic examination of washed and unwashed suspensions revealed no increase in overt acrosome loss during the $40 \mathrm{~min}$ after washing; by this time, fertilizing spermatozoa in the washed group would be expected to have initiated fusion with the egg plasma membrane (Fraser, 1983a). On the other hand, exposure of capacitated suspensions to sperm supernatants inhibited further acrosome loss in the sperm populations (Table 7). It would therefore appear that the inhibitory component stabilizes sperm membranes and inhibits the acrosome reaction. Its removal permits the spermatozoon to undergo the acrosome reaction, either in a directed manner as associated with egg penetration or in an undirected manner as seen in the timedependent general increase in proportions of acrosome-reacted cells in a population (e.g. Fraser, 1981, 1982; Dudenhausen \& Talbot, 1982).

There was also evidence that removal of the inhibitory component resulted in the expression of hyperactivated motility, an observation consistent with the increased fertility of washed samples. However, the exposure of capacitated suspensions to sperm supernatants had no detectable quantitative or qualitative effect on motility. Therefore, once this pattern has been stimulated, it appears to be irreversible, while the surface-related events associated with acrosome loss can still be inhibited.

The observation that supernatant-treated suspensions were unable to fertilize zona-intact eggs, despite the presence of many acrosome-free spermatozoa, suggests that fertilizing spermatozoa are drawn from the acrosome-intact population. That these same treated suspensions were able to fertilize zona-free eggs with no indication of inhibition suggests either that the acrosome-free spermatozoa retain their ability to fuse with the egg plasma membrane or that direct access to the egg surface is able to overcome the inhibition in acrosome-intact spermatozoa. The latter has some support in that unwashed suspensions tested after 30-min preincubation were poorly fertile with zona-intact eggs, yet were highly fertile and promoted a high incidence of polyspermy when mixed with zona-free eggs (Fraser, 1983a). In such suspensions, the proportion of acrosome-free spermatozoa is very low (Fraser, 1981, 1982), unlike the present capacitated suspensions which showed considerable acrosome loss (Table 7).

In all species examined, there is an obligatory requirement for $\mathrm{Ca}^{2+}$ to promote the acrosome reaction as well as sperm-egg interaction during fertilization (Yanagimachi, 1981). Since mouse spermatozoa require exposure to $\mathrm{Ca}^{2}+$ for at least the final 30-60 min of capacitation to attain full functional ability (Fraser, 1982), it seemed possible that loss of the inhibitory component might be associated with an increased permeability to calcium ions. In fact, both washing sperm suspensions preincubated in the absence of $\mathrm{Ca}^{2+}$ and exposure to hyperosmolal $\mathrm{Ca}^{2+}$-free media, with the subsequent introduction of exogenous $\mathrm{Ca}^{2+}$, resulted in a significant increase in fertilization rates over equivalent unwashed samples. This indicated that removal of the inhibitory component (demonstrated by the ability of the supernatants to inhibit fertilization by capacitated spermatozoa) did increase the response of spermatozoa to exogenous $\mathrm{Ca}^{2+}$. In comparison with equivalent washed control samples, however, these spermatozoa were still functionally deficient. The fact that inactivation of the inhibitory component was incomplete in $\mathrm{Ca}^{2+}$-free suspensions preincubated for $120 \mathrm{~min}$ (Table 5), compared with control suspensions, suggests that spermatozoa 
are actively involved in this process and that cells maintained in the absence of $\mathrm{Ca}^{2+}$ are functionally inadequate. Thus, the variability in fertility and rate of penetration exhibited by control suspensions preincubated for less than 120 min may reflect differences in the inactivation process in individual spermatozoa.

The present results are consistent with many earlier studies examining the effect of removal or addition of epididymal fluid to sperm suspensions. Although Gwatkin, Andersen \& Williams (1974) reported that washing mouse suspensions followed by preincubation removed fertilizing ability, no deleterious effect of washing was detected by Hoppe (1975) and Wolf, Hamada \& Inoue (1977) and a strain-dependent increase in fertility was noted by Fraser \& Drury (1976). In none of these was true rate of penetration assessed, however. An inhibitory effect on fertilization in vitro has been reported in numerous studies using homologous epididymal fluid and gametes (e.g. hamster: Morton \& Chang, 1973; mouse: Iwamatsu \& Chang, 1971; Oliphant \& Brackett, 1973; Fraser \& Drury, 1976; Wolf et al., 1977), with some evidence for cell surface association of the factor (Aonuma, Okabe, Kishi, Kawaguchi \& Yamada, 1982), and homologous or heterologous combinations of seminal plasma (s.p.). and gametes (e.g. rabbit s.p. and rat; Davis \& Niwa, 1974; rabbit s.p. and rabbit: Gould, Srivastava, Cline \& Williams, 1971; human s.p. and human/hamster: Kanwar, Yanagimachi \& Lopata, 1979; human s.p. and mouse: Reddy, Stark \& Zaneveld, 1979). In most of these studies the mechanism of inhibitory action was not examined, although Reddy et al. (1979) suggested that acrosome loss was not inhibited because treated sperm suspensions were able to fertilize zona-free eggs despite their inability to fertilize zona-intact eggs. There was no inhibition of the guinea-pig acrosome reaction by the inhibitory factor purified from human seminal plasma (Reddy, Audhya, Goodpasture \& Zaneveld, 1982). Despite this, the majority of investigations looking at possible interaction between male reproductive tract fluids and the acrosome (Davis \& Niwa, 1974; Bavister, Rogers \& Yanagimachi, 1978; Eng \& Oliphant, 1978; Fleming \& Wai, 1978; Hyne \& Garbers, 1982) have indicated an inhibition of the acrosome reaction, findings consistent with those in the present study.

The apparent similarity in action of these inhibitory substances does not imply that the same molecular species are involved, however. The active component from porcine epididymal fluid is reported to be of low molecular weight (Hyne \& Garbers, 1982), while those from human (Kanwar et al., 1979; Reddy et al., 1979) and rabbit (Davis \& Niwa, 1974; Eng \& Oliphant, 1978) seminal plasma appear to be of high molecular weight. Preliminary (unpublished) results suggest that the inhibitory component examined in the present study is either macromolecular or attached to a macromolecular species. The present study has shown that (1) epididymal mouse spermatozoa have an inhibitory component associated with the cell surface, (2) during incubation under capacitating conditions this component is destroyed or inactivated and (3) a primary action is inhibition of the acrosome reaction. It is proposed that this represents a general mechanism common to capacitation in all mammalian species, with species-dependent variation in inhibitor-sperm cell affinity and ease of inhibitor inactivation or destruction.

I thank Dr R. A. P. Harrison for many stimulating discussions and Ms Jennifer Small for preparation of the samples for electron microscopy. This work was supported in part by grants from the Marie Stopes Research Fund and the Agricultural Research Council.

\section{References}

Aonuma, S., Okabe, M., Kishi, Y., Kawaguchi, M. \& Yamada, H. (1982) Capacitation inducing activity of serum albumin in fertilization of mouse ova in vitro. $J$. Pharm. Dyn. 5, 980-987.

Austin, C.R. (1951) Observations on the penetration of the sperm into the mammalian egg. Aust. J.sci. Res. B 4, 581-596.
Bavister, B.D., Rogers, B.J. \& Yanagimachi, R. (1978) The effects of cauda epididymal plasma on the motility and acrosome reaction of hamster and guinea pig spermatozoa in vitro. Biol. Reprod. 19, 358-363.

Bedford, J.M. (1970) Sperm capacitation and fertilization in mammals: Biol. Reprod., Suppl. 2, 128-158. 
Bedford, J.M. \& Chang, M.C. (1962) Removal of decapacitation factor from seminal plasma by high speed centrifugation. Am. J. Physiol. 202, 179-181.

Brackett, B.G. \& Oliphant, G. (1975) Capacitation of rabbit spermatozoa in vitro. Biol. Reprod. 12, 260-274.

Chang, M.C. (1951) Fertilizing capacity of spermatozoa deposited into the Fallopian tubes. Nature, Lond. 168, 697-698.

Chang, M.C. (1957) A detrimental effect of rabbit seminal plasma on the fertilizing capacity of sperm. Nature, Lond. 179, 258-259.

Davis, B.K. \& Niwa, K. (1974) Inhibition of mammalian fertilization in vitro by membrane vesicles from seminal plasma. Proc. Soc. exp. Biol. Med. 16, 11-16.

Dudenhausen, E. \& Talbot, P. (1982) Detection and kinetics of the normal acrosome reaction of mouse sperm. Gamete Res. 6, 257-265.

Eng, L.A. \& Oliphant, G. (1978) Rabbit sperm reversible decapacitation by membrane stabilization with a highly purified glycoprotein from seminal plasma. Biol. Reprod. 19, 1083-1094.

Fleming, A. \& Wai, P. (1978) Effect of whole and fractionated epididymal fiuid on acrosome reaction and fertilization. Biol. Reprod. 18, Suppl. 1, Abstr. $77 \mathrm{~A}$.

Fraser, L.R. (1977) Motility patterns in mouse spermatozoa before and after capacitation. J. exp. Zool. 202, 439-444.

Fraser, L.R. (1981) Dibutyryl cyclic AMP decreases capacitation time in vitro in mouse spermatozoa. $J$. Reprod. Fert. 62, 63-72.

Fraser, L.R. (1982) $\mathrm{Ca}^{2+}$ is required for mouse sperm capacitation and fertilization in vitro. J. Androl. 3, 412-419.

Fraser, L.R. (1983a) Mouse sperm capacitation assessed by kinetics and morphology of fertilization in vitro. $J$. Reprod. Fert. 69, 419-428.

Fraser, L.R. (1983b) Potassium ions modulate expression of mouse sperm fertilizing ability, acrosome reaction and hyperactivated motility in vitro. J. Reprod. Fert. 69, 539-553.

Fraser, L.R. \& Drury, L.M. (1976) Effect of removal of epididymal secretions on fertilization in vitro of mouse eggs. J. Reprod. Fert. 48, 125-128.

Gould, K.G., Srivastava, P.N., Cline, E.M. \& Williams, W.L. (1971) Inhibition of in vitro fertilization of rabbit ova with naturally occurring antifertility agents. Contraception 3, 261-267.

Gwatkin, R.B.L., Andersen, O.F. \& Williams, D.T. (1974) Capacitation of mouse spermatozoa in vitro: involvement of epididymal secretions and cumulus oophorus. J. Reprod. Fert. 41, 253-256.
Hoppe, P.C. (1975) Fertilizing ability of mouse sperm from different epididymal regions and after washing and centrifugation. J. exp. Zool. 192, 219-222.

Hyne, R.V. \& Garbers, D.L. (1982) Inhibition of the guinea-pig sperm acrosome reaction by a low molecular weight factor(s) in epididymal fluid and serum. J. Reprod. Fert. 64, 151-157.

Iwamatsu, T. \& Chang, M.C. (1971) Factors involved in the fertilization of mouse eggs in vitro. J. Reprod. Fert. 26, 197-208.

Johnson, M.H. (1975) The macromolecular organization of membranes and its bearing on events leading up to fertilization. J. Reprod. Fert. 44, 167-184.

Kanwar, K.C., Yanagimachi, R. \& Lopata, A. (1979) Effects of human seminal plasma on fertilizing capacity of human spermatozoa. Fert. Steril. 31, 321327.

Morton, B. \& Chang, T.S.K. (1973) The effect of fluid from the cauda epididymidis, serum components and caffeine upon the survival of diluted epididymal hamster spermatozoa. J. Reprod. Fert. 35, 255-263.

Oliphant, G. \& Brackett, B.G. (1973) Capacitation of mouse spermatozoa in media with elevated ionic strength and reversible decapacitation with epididymal extracts. Fert. Steril. 24, 948-955.

O'Rand, M.G. (1979) Changes in sperm surface properties correlated with capacitation. In The Spermatozoon, pp. 195-204. Eds D. W. Fawcett \& J. M. Bedford. Urban and Schwarzenberg, Baltimore.

O'Rand, M.G. (1982) Modification of the sperm membrane during capacitation. Ann. N.Y. Acad. Sci. 383, 392-404.

Reddy, J.M., Stark, R.A. \& Zaneveld, L.J.D. (1979) A high molecular weight antifertility factor from human seminal plasma. J. Reprod. Fert. 57, 437-446.

Reddy, J.M., Audhya, T.K., Goodpasture, J.C. \& Zaneveld, L.J.D. (1982) Properties of a highly purified antifertility factor from human seminal plasma. Biol. Reprod. 27, 1076-1083.

Snedecor, G. \& Cochran, W. (1967) Statistical Methods, 6th edn. Iowa State University Press, Ames.

Wolf, D.P., Hamada, M. \& Inoue, M. (1977) Kinetics of sperm penetration into and the zona reaction of mouse ova inseminated in vitro. J. exp. Zool. 201, 2936.

Yanagimachi, R. (1970) The movement of golden hamster spermatozoa before and after capacitation. J. Reprod. Fert. 23, 193-196.

Yanagimachi, R. (1981) Mechanisms of fertilization in mammals. In Fertilization and Embryonic Development, pp. 81-182. Eds L. Mastroianni \& J. D. Biggers. Plenum Publishing, New York. 